\section{When order comes naturally}

\author{
Self-Organization in Biological \\ Systems \\ by Scott Camazine, Jean-Louis \\ Deneubourg, Nigel R. Franks, James Sneyd, \\ Guy Theraulaz \& Eric Bonabeau \\ Princeton University Press: 2001.535 pp. \\ $\$ 65, £ 43$
}

\section{Eshel Ben-Jacob}

An old man is sitting by a pile of rocks, carving one into a block. A passer-by asks him what he is doing. "Can't you see?" says the old man. "I'm building a cathedral." What would a mound-building termite, carrying a ball of mud, reply? Does it know that it is taking part in the construction of one of nature's most astounding structures? The authors of Self-Organization in Biological Systems hold the belief that the termites in the colony don't have a notion of participating in a group task. The mound, they say, emerges through a process of self-organization, defined as "a property of certain dynamical mechanisms whereby structures, patterns and decisions appear at the global (colony) level of a system, based on interactions among its lower-level components (agents, individuals)". They favour the view that the process is executed on the basis of purely local information, without reference to the global level.

In the first part of the book, the authors describe self-organization and define relevant concepts, such as pattern, complexity and emergence. They explain how self-organization works, with emphasis on the role of positive and negative feedback, amplification of fluctuations, local information and information transfer between individuals. Self-organizing systems are characterized, modelling approaches discussed and alternative mechanisms presented.

Next, the authors address their primary goal: "to demonstrate, for a wide range of examples, the link between the rather simple behavioural programs of the individuals in a group and the sophisticated structures and patterns that emerge from their collective activity." With present computational power, it is only natural to use computer models to study self-organization. In physics and chemistry, models have long served as an indispensable research tool. But in the life sciences they have a different role, and many biologists still question the value and predictive power of models. This book should contribute to a recognition of the usefulness of modelling in the study of biocomplexity.

But simulations alone, without analysis, are insufficient. One can easily fall into the 'reminiscence trap', described by J. D. Cowan as the tendency to devise a set of rules that will

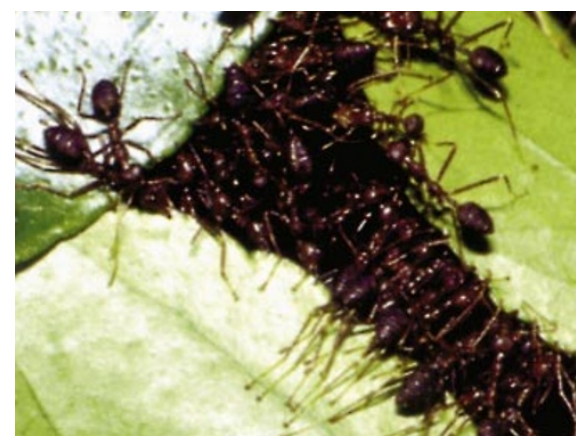

Group participation: worker ants joining leaves (top), and the spiral organization of a daisy head.

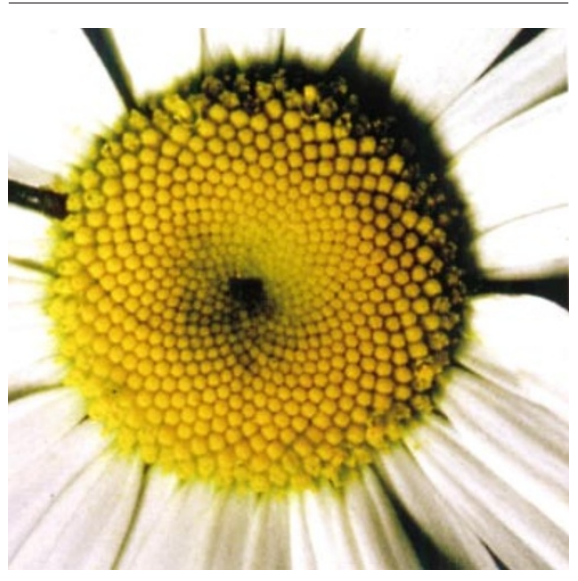

just mimic aspects of observed phenomena. But this can be prevented if one understands the fundamental mechanisms at their heart. Then, a model can serve to make reliable predictions, and not just reproduce existing behaviour. The desire to achieve this naturally pushes one in the direction of including more biological details. Nevertheless, the other extreme must also be avoided — the 'realistic trap' - where the model becomes swamped with too many details (and, usually, unknown parameters) and hence loses all predictive power. Model building, indeed, is "an art in its own right" (the skills can only be acquired by practice), the challenge being how to elicit the generic features and basic principles needed to explain behaviour from experimental observations and biological knowledge.

Many interesting examples of biological self-organization are presented — from complex patterning of bacterial colonies through mound-building termites and other social insects to schools of fish. In each of the case studies, the transition from observation to modelling is demonstrated, along with model analysis, simulations and comparison with the observed phenomena, in which the authors take the reader, step-by-step, through the construction of the model.

Although local interactions are undoubtedly important, I don't agree with the authors that they are sufficient to explain bioself-organization. For example, even simple microorganisms can gather information about the global state of their colony. It is known that bacteria chatter via chemical signals that propagate throughout the colony, thus providing each bacterium with global-level information. For example, some bacterial strains sporulate collectively when starved, but each bacterium does so only after broadcasting its starvation and receiving an echo - in the form of a chemical message that the entire colony is ready to sporulate.

When studying self-organization and pattern formation in non-living systems, the questions asked are largely of a mechanistic nature. But living systems pose another question that is far more challenging — that of origin. The authors believe the evidence they have amassed in this book corroborates what has long been an automatic answer to this question - that "much of the complexity of self-organised structures seen in biology arises because the rules governing the interactions among the components of biological systems have evolved through natural selection". They raise this issue throughout the book, but I was not convinced, as on the basis of the very same information one may just as well arrive at the opposite conclusion - that self-organization and natural selection are two alternative evolutionary mechanisms. The authors do mention that some biologists hold the belief that the first is an alternative to the second, which they glibly dub a "misconception". But when the same set of facts can lead to two opposite conclusions, the reason might be that neither is really a conclusion. This reservation aside, the book unfolds, in a very interesting and useful manner, the body of work relating to the mechanisms involved in biological self-organization.

Eshel Ben-Jacob is in the School of Physics,

Tel Aviv University, 69978 Tel Aviv, Israel.

\section{More on self-organization}

\section{Emergence: The Connected Lives of} Ants, Brains, Cities and Software

by Steven Johnson

Allen Lane/Scribner, $£ 14.99 / \$ 25$

correction In the review by Howard P. Segal of IBM and the Holocaust: The Strategic Alliance Between Nazi Germany and America's Most Powerful Corporation by Edwin Black (Nature 411, 993-994; 2001), one paragraph stated: "Polish ghetto exterminators ... never had punch-card machines, and the SS Race and Settlement Office obtained them only in 1943." In fact, the SS Race and Settlement Office was an SS officers' marriage and adoption-screening bureau, not a prime mover in the extermination process.

The review also stated: "Black minimizes the inefficiency of many Nazi procedures, which, for example, delayed many trainloads of Jews en route to concentration camps." In fact, custom-designed IBM programs helped the Nazis to locate and deploy the locomotives and boxcars. Virtually all European trains were at that time tracked by Hollerith cards.

These small errors do not affect the rest of the review, or its general conclusions. 